\title{
On The Effectiveness of Probiotic as Treatment Strategy of Autism
}

\author{
Afaf El-Ansary ${ }^{1-4 *}$ and Naima Zayed ${ }^{4}$ \\ ${ }^{1}$ Central laboratory, Female Centre for Scientific and Medical Studies, King Saud University, Saudi Arabia \\ ${ }^{2}$ Autism Research and Treatment Centre, Riyadh, Saudi Arabia \\ ${ }^{3}$ Member in Council for Nutritional and Environmental Medicine (CONEM) Norway and Head of CONEM, Child Brain Research Group, Saudi Arabia \\ ${ }^{4}$ Therapeutic Chemistry Department, National Research Centre, Dokki, Cairo, Egypt \\ *Corresponding author: Afaf El-Ansary, Central laboratory, Female Centre for Scientific and Medical Studies, Autism Research and Treatment Centre, \\ Riyadh, Member in Council for Nutritional and Environmental Medicine (CONEM) Norway and Head of CONEM Saudi Child Brain Research Group, \\ King Saud University, Saudi Arabia
}

Submission: April 01, 2018; Published: April 17, 2018

\begin{abstract}
Abbreviations: SCFAs: Short Chain Fatty Acids; LPS: Lip Polysaccharides; TNF- $\alpha$ : Tumor Necrosis Factor- $\alpha$; IL-6: Inter leukin-6; TNF- $\alpha$ : Tumor Necrosis
\end{abstract} Factor- $\alpha$; BBB: Blood Brain Barrier; GABA: Gamma Amino Butyric Acid; GI: Gastro Intestinal

\section{Introduction}

The gut micro biota and its metabolites play important roles in the body physiology, including the brain. Dysbiosis or micro biota alterations are involved in numerous pathologies, including neuro developmental disorders. The frequent occurrence of gastrointestinal symptoms in autistic patients could suggest the possible involvement of impaired gut micro biota as etiological mechanism in autism. Up to our understanding of the etiological mechanisms of autism through our clinical and experimental research, it was interesting to relate altered gut micro biota to neuro inflammation, oxidative stress, heavy metal toxicity, autoimmunity, and glutamate excitotoxicity to clarify the possibility of targeting gut micro biota to ameliorate these contributed pathologic signaling and thus treat autism. The gut micro biota can indirectly affect the brain through the circulating pro-inflammatory and antiinflammatory cytokines or through the production of metabolites among which is short chain fatty acids (SCFAs). SCFAs such as acetate, butyrate, and propionate can modulate the innate immune system and the sympathetic nervous system [1]. Alteration in the intestinal bacteria is related to reduced integrity of the intestinal barrier, leading to increased absorption of toxic metabolites from the gut and leakage of lip polysaccharides (LPS) and fatty acids. These toxic molecules can activate systemic inflammation [2], and finally affects the brain. Multiple records indicate that neuro inflammation biomarkers, such as Inter leukin-6 (IL-6) and Tumor Necrosis Factor- $\alpha$ (TNF- $\alpha$ ) are greatly involved as etiological mechanism in autism [3].

Heberling et al. [4] hypothesized that overgrowth of Clostridia and/or Desulfovibrio and a decrease in Bifidobacterium which is involved in autism pathogenesis can be related to oxidative stress and impaired sulfur metabolism. Together with increased intestinal permeability, disruption of the blood brain barrier (BBB), increased blood circulation of bacterial metabolites and toxins can induce neuro inflammation. Since, autistic patients are poor detoxifiers and they have a burden of lead and mercury levels, it was very interesting to discuss the probiotic-mediated detoxification mechanism [5-7]. Comparative study on conventional against germ-free animals have shown the protective role of gut micro biota against mercury and lead intoxication [8]. This natural protective effect can clearly have demonstrated through the remarkable increase of the probiotic lactobacilli in response to heavy metal [9]. Although certain pathogenic bacteria such as Clostridia and Desulfovibrio as methyl atorscan convert the less toxic organic mercury into the more toxic methyl mercury [10-12], there are certain probiotic strains that have active enzymatic pathways for detoxification, such as mercury reduction and demethylation [13].

Glutamate release, uptake, metabolism and signaling disorders are described to contribute to the etiology of autism [14-17]. Dysregulation of the enteric glutamatergic neurotransmitter is also among the several pathological mechanisms related to autism. Up to molecular mimicry between early life over grown micro biota and human proteins, auto antibodies against the receptors of the Gamma Amino Butyric Acid (GABA), as well as important immunerelated enzymes such as transglutaminase- 2 can be related to glutamate excitotoxicity. Up to Heberling et al. [4] hypothesis relating oxidative stress, impaired sulfur metabolism, overgrowth of pathogenic bacteria and increased gut permeability to each 
other as four etiological mechanisms in autism, it was suggested that all these mechanisms should be targeted as treatment strategy. Combining folinic acid, betaine, and methyl vitamin B12, as treatment of impaired sulfur metabolism, vancomycin as treatment to clostridia overgrowth, aztreonam as treatment of Desulfovibrio overgrowth, zonulin as treatment of loosening of tight junctions could have great potential in treating autism symptoms [18-20].

Consumption of probiotics is now being recommended as an adjuvant for detoxification in patients with autism. In relation to methyl mercury burden in autistic patients, lower Bifidobacterium spp. [21] and clostridia overgrowth [22], again Lactobacillus spp. and Bifidobacterium spp. as probiotic species able to transform toxic mercury compounds into metabolites extractable in feces [23]. Interestingly, selected strains of probiotics have been shown to inhibit the growth of different Clostridium species in vitro [24] and in vivo [25]. Consumption of probiotics may therefore help to maintain or restore the balance of gut micro biota among autistic children [26]. Moreover, Lactobacillus rhamnosus was found to reduce anxiety and depression-like behaviors through the amelioration GABAergic system of the brain [27]. Moreover oral administration of GABA derived from Lactobacillus hilgardii fermentation has been shown to have clinical value presented as immune enhancement and anxiety reduction [28].

In relation to all these alterations, Santocchi et al. [29] registered a randomized clinical trial (NCT02708901) on 100 preschooler's autistic patients (18-72 months age) with and without gastro Intestinal (GI) symptoms. Vivomixx $\AA$ as a probiotic mixture of 8 bacterial strains (Bifidobacteriumlongum, Bifidobacteriuminfantis, Bifidobacteriumbreve, Lactobacillus plantarum, Lactobacillus acidophilus, Lactobacillus paracasei, Lactobacillus bulgaricus and Streptococcus thermophilus) was fed for both groups for six months. Both groups of participants were assessed at the baseline, after three months and after six months from the baseline to evaluate the possible changes in GI symptoms, in autism behavioral phenotypes (e.g. social communication; sensory profile), plasmatic, urinary and fecal biomarkers related to abnormal intestinal function and in the electrophysiological patterns. The results of this trial are about to be declared in 2018 .

\section{Conclusion}

At this point, the role of the gut micro biota as a trigger and modulator of heavy metal toxicity, neuro inflammation, autoimmunity, and glutamate excitotoxicity as etiological mechanisms of autism remains to be formally ascertained. Identification of pathogenic overgrowth of flora (individually or all together) may give new diagnostic tools. More importantly and up to this, new therapeutic strategies could be designed to neutralize the bacterial pathogenic triggers (e.g. bacterial neuro peptide, toxin and metabolites). These would include antimicrobial manipulation by specifically tailored antibiotic protocols, by pre and probiotics. Figure 1 demonstrates the suggested therapeutic effects of probiotics through the amelioration of oxidative stress, neuro inflammation, heavy metal toxicity, glutamate excitotoxicity and autoimmunity. Of course, addressing these etiological mechanisms can improve attention, learning and other autistic behaviors.

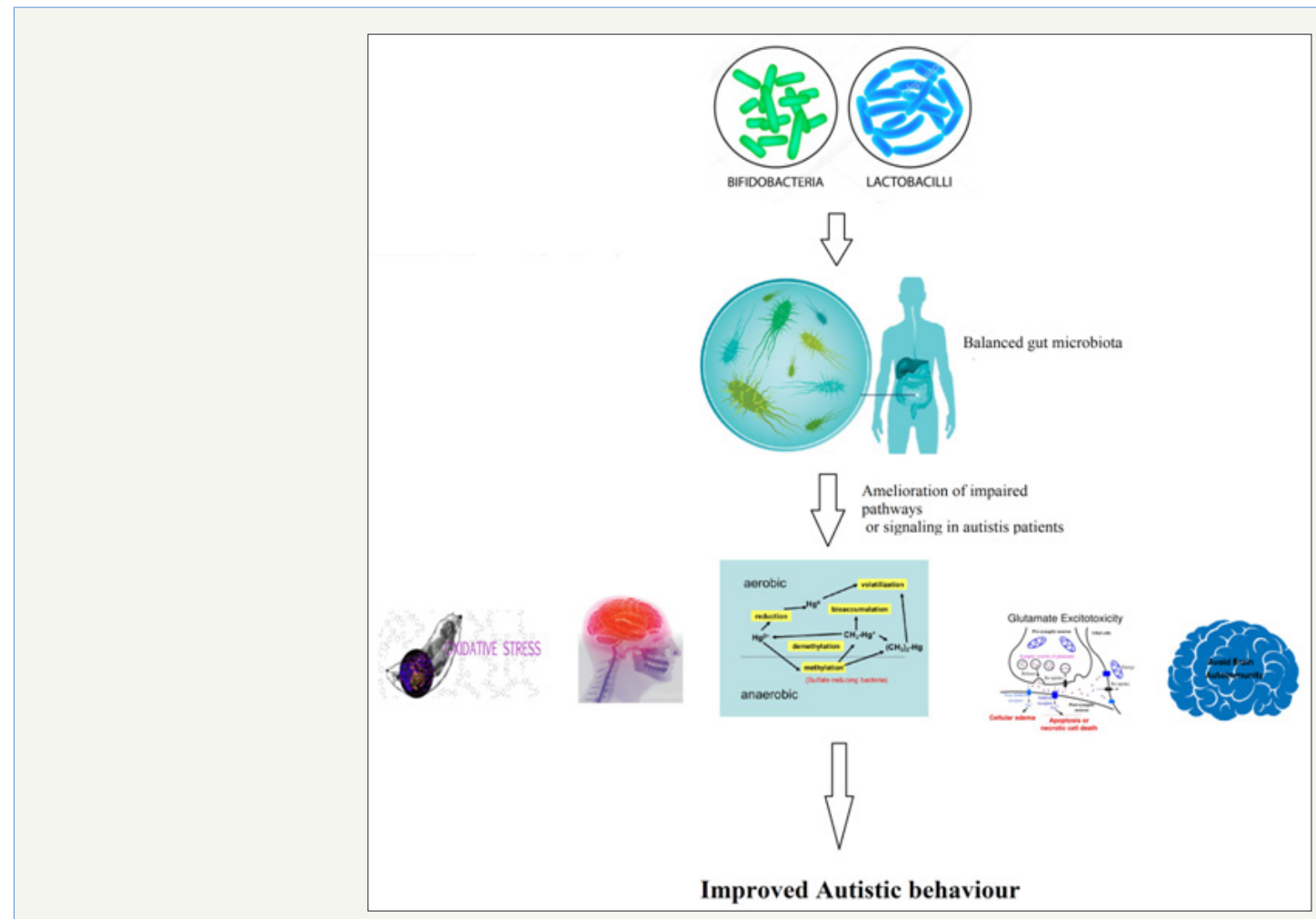

Figure 1: Suggested therapeutic effects of probiotics through the amelioration of oxidative stress, neuro inflammation, heavy metal toxicity, glutamate excitotoxicity, and autoimmunity in autistic patients. 


\section{References}

1. Borre YE, O Keeffe GW, Clarke G, Stanton C, Dinan TG, et al. (2014) Microbiota and neurodevelopmental windows: implications for brain disorders. Trends Mol Med 20(9): 509.

2. Theoharides TC, Asadi S, Patel A (2013) Focal brain inflammation and autism. J Neuro inflammation 10: 46.

3. Khailova L, Dvorak K, Arganbright KM, Halpern MD, Kinouchi T (2009) Bifidobacteriumbifidum improves intestinal integrity in a rat model of necrotizing enterocolitis. Am J Physiol Gastrointest Liver Physiol 297(5): G940-G949.

4. Heberling CA, Dhurjati PS, Sasser M (2013) Hypothesis for a systems connectivity model of Autism Spectrum Disorder pathogenesis: links to gut bacteria, oxidative stress, and intestinal permeability. Med Hypotheses 80(3): 264-270.

5. Alabdali A, Al-Ayadhi L, El-Ansary A (2014) A key role for an impaired detoxification mechanism in the etiology and severity of autism spectrum disorders. Behav Brain Funct 10: 14.

6. El-Ansary AK, Ben Bacha A, Kotb M (2012) Etiology of autistic features: the persisting neurotoxic effects of propionic acid. J Neuroinflammation 9. 74 .

7. Tian F, Zhai Q, Zhao J, Liu X, Wang G (2012) Lactobacillus plantarum CCFM8661 alleviates lead toxicity in mice. Biol Trace Elem Res 150(13): 264-271.

8. Claus SP, Guillou H, Ellero-Simatos S (2016) The gut microbiota: a major player in the toxicity of environmental pollutants? npj Biofilms and Microbiomes 16003.

9. Breton J, Massart S, Vandamme P, De Brandt E, Pot B, et al. (2013) Ecotoxicology inside the gut: impact of heavy metals on the mouse microbiome. BMC Pharmacol Toxicol 14: 62

10. Limper U, Knopf B, König H (2008) Production of methyl mercury in the gut of the Australian termite Mastotermes darwiniensis. J Appl Entomol 132(2): 168.

11. Rothenberg SE, Keiser S, Ajami NJ, Wong MC, Gesell J, et al. (2016) The role of gut micro biota in fetal methylmercury exposure: Insights from a pilot study. Toxicol Lett 242: 60-62.

12. Ma M, Du H, Wang D, Kang S, Sun T (2017) Biotically mediated mercury methylation in the soils and sediments of Nam Co Lake, Tibetan Plateau. Environ Pollut 227: 243-251.

13. Bisanz JE, Enos MK, Mwanga JR, Changalucha J, Burton JP, et al. (2014) Randomized open-labelpilot study of the influence of probiotics and the gutmicrobiome on toxic metal levels in Tanzanian pregnant women and school children. MBio 5(5): e01580-14.

14. Miladinovic T, Nashed MG, Singh G (2015) Overview of glutamatergic dysregulation in central pathologies. Biomolecules 5(4): 3112-3141.

15. El-Ansary A, Al-Ayadhi L (2014) GABAergic/glutamatergic imbalance relative to excessive neuroinflammation in autism spectrum disorders. J Neuroinflammation 11: 189.

16. Al-Otaish H, Al-Ayadhi L, Bjørklund G, Chirumbolo S, El-Ansary A (2018) Relationship between absolute and relative ratios of glutamate, glutamine and GABA and severity of autism spectrum disorder. Metab Brain Dis.

17. Hamed N, Al-Ayadhi L, Mohamed O, Abdalla E, Hanan Q et al. (2018) Understanding the roles of glutamine synthetase, glutaminase, and glutamate decarboxylase autoantibodies in imbalanced excitatory/ inhibitory neurotransmission as etiological mechanisms of autism. Psychiatry Clin Neurosci.

18. Finegold SM (2008) Therapy and epidemiology of autism-clostridial spores as key elements. Med Hypotheses 70(3): 508-511.

19. Finegold SM (2011) Desulfovibrio species are potentially important in regressive autism. Med Hypotheses 77(2): 270-274.

20. Watts T, Berit I, Sapone A, Gerarduzzi T, Not T (2005) Role of the intestinal tight junction modulator zonulin in the pathogenesis of type I diabetes in BB diabetic-prone rats. Proc Natl Acad Sci USA 102(8): 2916-2921.

21. Adams JB, Johansen LJ, Powell LD, Quig D, Rubin RA (2011) Gastrointestinal flora and gastrointestinal status in children with autism-comparisons to typical children and correlation with autism severity. BMC Gastroenterol 11(1): 22.

22. Song Y, Liu C, Finegold SM (2004) Real-time PCR quantitation of clostridia in feces of autistic children. Appl Environ Microbiol 70(11): 6459-6465.

23. Brudnak MA (2002) Probiotics as an adjuvant to detoxification protocols. Med Hypotheses 58(5): 382-385

24. Bogovič Matijašić B, Koman RM, Perko B, Rogelj I (2007) Inhibition of Clostridium tyrobutyricum in cheese by Lactobacillus gasseri. Int Dairy J 17(2): 157-166.

25. Mc Farland LV (2009) Evidence-based review of probiotics for antibioticassociated diarrhea and Clostridium defficile infections. Anaerobe 15(6): 274-280

26. Fooks L, Gibson G (2002) Probiotics as modulators of the gut flora. Br J Nutr 88(1): 39.

27. Bravo JA, Forsythe P, Chew MV, Escaravage E, Savignac HM, et al. (2011) Ingestion of Lactobacillus strain regulates emotional behavior and central GABA receptor expression in a mouse via the vagus nerve. Proc Natl Acad Sci USA 108(38): 16050-16055.

28. Abdou AM, Higashiguchi S, Horie K, Kim M, Hatta H, (2006) Relaxation and immunity enhancement effects of gamma-aminobutyric acid (GABA) administration in humans. Biofactors 26(3): 201-208.

29. Santocchi E, Guiducci L, Fulceri F, Billeci L, Buzzigoli E, et al. (2016) Gut to brain interaction in Autism Spectrum Disorders: a randomized controlled trial onthe roleof probiotics on clinical, biochemical and neuro physiological parameters. BMC Psychiatry 16: 183. 
Creative Commons Attribution 4.0 International License

For possible submissions Click Here

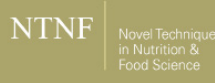

Submit Article
Novel Techniques in Nutrition and Food Science

\section{Benefits of Publishing with us}

- High-level peer review and editorial services

- Freely accessible online immediately upon publication

- Authors retain the copyright to their work

- Licensing it under a Creative Commons license

- Visibility through different online platforms 